\title{
Core-shell lipid polymer nanoparticles for combined chemo and gene therapy of childhood head and neck cancers
}

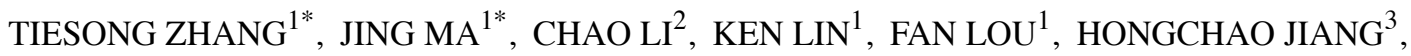 \\ YINGQIN GAO ${ }^{1}$, YANLI YANG ${ }^{4}, \mathrm{CHENG} \mathrm{MING}^{5}$ and BIAO RUAN ${ }^{4}$ \\ Departments of ${ }^{1}$ Otolaryngology, Head and Neck Surgery, ${ }^{2}$ Anesthesiology and ${ }^{3}$ Clinical Laboratory, \\ Kunming Children's Hospital; ${ }^{4}$ Department of Otolaryngology, The First Affiliated Hospital of Kunming Medical University; \\ ${ }^{5}$ Department of Otolaryngology, The Third People's Hospital of Yunnan Province, Kunming, Yunnan 650032, P.R. China
}

Received March 20, 2016; Accepted August 10, 2016

DOI: $10.3892 / o r .2017 .5365$

\begin{abstract}
Pediatric head and neck cancers account for overall $12 \%$ of all pediatric cancers. Despite recent advances in therapeutic modalities, children with tumor metastasis have poor prognosis. Therefore, there is an unmet need for new and effective treatment modalities for pediatric head and neck cancers. The present study describes a simple and efficient method for fabrication of cationic lipid-polymer hybrid nanoparticles (CLPNs) for co-delivery of cisplatin (CDDP) and DNA (CDDP/DNA CLPNs) for the therapy of childhood head and neck cancers. CDDP/DNA CLPNs were prepared by the modified double emulsion solvent evaporation method with self-assembly. CDDP-loaded CLPNs (CDDP CLPNs), CDDP-loaded polymeric nanoparticles (PNPs) (CDDP PNPs), and DNA-loaded Lipofectamine ${ }^{\circledR} 2000$ (DNA LIPO) were also prepared for comparison. The results illustrated that the concentration of the cationic lipid has influence on the characteristics of CLPNs. In vitro anticancer effect, in vitro transfection efficiency, in vivo antitumor and gene delivery efficacy of CDDP/DNA CLPNs have advantages over other formulations tested. In conclusion, outstanding delivery ability of CLPNs for both CDDP and DNA could combine the therapeutic efficiency of both drug and gene for the treatment of pediatric rhabdomyosarcoma (RMS).
\end{abstract}

\section{Introduction}

Pediatric malignancies are the leading cause of disease-related death in children and the second overall cause of death

Correspondence to: Dr Biao Ruan, Department of Otolaryngology, The First Affiliated Hospital of Kunming Medical University, No. 295 Xichang Road, Kunming, Yunnan 650032, P.R. China E-mail: rbynent@163.com

*Contributed equally

Key words: pediatric head and neck cancers, cisplatin, gene therapy, cationic lipid-polymer hybrid nanoparticles, rhabdomyosarcoma after accidents (1). Pediatric head and neck cancers account for overall $12 \%$ of all pediatric cancers (2). Recently, pediatric head and neck cancers were shown to increase faster than pediatric cancers overall. Lymphoma, particularly non-Hodgkin lymphoma, rhabdomyosarcoma (RMS), and nasopharyngeal carcinoma (NPC) are the most common pediatric cancers of the head and neck region $(3,4)$. Among them, RMS is the most common soft-tissue sarcoma found in children and adolescents (5). The currently available treatments include surgery, chemotherapy, and radiation. In view of their high metastatic potential, chemotherapy has become one of the main treatments recently. Despite recent advances in therapeutic modalities, children with tumor metastasis have poor prognosis. Therefore, there is an unmet need for new and effective treatment modalities for pediatric head and neck cancers.

Platinum-based agents form the backbone of the standard chemotherapeutic regimens for head and neck cancers of both children and adults. Specifically, cisplatin (CDDP) is the most commonly used drug in the treatment of RMS and NPC, the two most common pediatric cancers of the head and neck region $(6,7)$. However, CDDP is nephrotoxic with the toxicity profile increasing with increase in dose; development of drug resistance and several dose-limiting toxicities have severely led to poor patient compliance and failure of chemotherapy $(8,9)$. Therefore, any strategy that would reduce the dose and toxicity of CDDP-based chemotherapy consequently is a welcome measure in oncology practice.

The combination of CDDP with other therapeutic methods, such as gene therapy, has become an attractive treatment project for head and neck cancers $(10,11)$. Nanocarrier-based chemotherapeutic drugs, either alone or in combination with gene anticancer therapies, are currently under development with the goal of improving survival and clinical outcome (12-14). Among the various non-viral gene vectors, cationic lipids are ideal gene carriers because of their superior transfection efficiency, excellent gene-incorporation ability (15). Cationic lipid-polymer hybrid nanoparticles (CLPNs), which may take advantage of the unique strengths of both polymeric nanoparticles (PNPs) and liposomes, are the ideal carriers for co-delivery of chemotherapeutics and genes (16-19). CLPNs are core-shell nanoparticle structures comprising polymer cores and lipid 
layers (20). Recently, LPNs of CDDP with high drug-loading capability were investigated and showed excellent safety and antitumor efficacy in mice $(14,21)$. Nano-combination delivery systems of CDDP with DNA may reverse drug resistance and hence enhance the efficacy of CDDP to eventually improve overall treatment outcomes.

The present study describes a simple and efficient method for fabrication of CLPNs for co-delivery of CDDP and DNA (CDDP/DNA CLPNs). Plasmid-enhanced green fluorescent protein was selected as the model DNA. CDDP-loaded CLPNs (CDDP CLPNs), CDDP-loaded PNPs (CDDP PNPs), and DNA-loaded Lipofectamine ${ }^{\circledR} 2000$ (DNA LIPO) were also prepared for comparison. The influence of cationic lipid concentration on the characteristics of CLPNs was evaluated. In vitro anticancer effect of free drug and CLPNs, and in vitro transfection efficiency were investigated on human RMS cell line, RD-4 cells. Finally, in vivo antitumor efficacy study was performed in the xenograft tumor model.

\section{Materials and methods}

Materials. CDDP, PLGA (50:50) [molecular weight (MW), 7,000-17,000], poly(vinyl alcohol) (PVA) (87-89\% hydrolyzed; MW, 13,000-23,000), 3-(4,5-dimethylthiazol-2-yl)-2,5-diphenyltetrazolium bromide (MTT) were purchased from Sigma-Aldrich (Shanghai, China). Plasmid EGFP (pEGFP) was obtained from Clontech Laboratories, Inc. (Palo Alto, CA, USA). Cationic lipid 1,2-di-(9Z-octadecenoyl)-3-trimethylammonium-propane (DOTAP) was purchased from Avanti Polar Lipids (Alabaster, AL, USA). Lipofectamine ${ }^{\circledR} 2000$ Transfection Reagent was obtained from Thermo Fisher Scientific(Waltham,MA,USA).Quant-iT ${ }^{\mathrm{TM}}$ PicoGreen ${ }^{\circledR} \mathrm{dsDNA}$ quantitation reagent was obtained from Invitrogen by Life Technologies (Carlsbad, CA, USA). Fetal bovine serum (FBS) was the product of Thermo Fisher Scientific (Fairlawn, NJ, USA). All other chemicals were of analytical grade and used as obtained without further purification.

Preparation of CDDP/DNA CLPNS and CDDP CLPNS. CDDP/DNA CLPNs were prepared by the modified double emulsion solvent evaporation method with self-assembly (Fig. 1) (18). Briefly, CDDP (0.1 g), PLGA (0.3 g), and DOTAP were dissolved in dichloromethane $(10 \mathrm{ml})$ to form the organic phase. pEGFP $(0.1 \mathrm{~g})$ was dissolved in an aqueous solution $(1 \mathrm{ml})$. The water-in-oil emulsion was formed by the addition of an aqueous phase into an organic phase with sonication. The primary emulsion was transferred to $1 \% \mathrm{w} / \mathrm{v}$ PVA aqueous solution and sonicated in an ice bath. The resultant secondary (water-in-oil-in-water) emulsion was stirred overnight at room temperature until the evaporation of dichloromethane was complete. The final formulated CDDP/DNA CLPNs were washed three times by a repeating centrifugation step and freeze-dried at $-20^{\circ} \mathrm{C}$. To investigate the influence of cationic lipid concentrations on size, charge, and in vitro performance, five formulation groups of CDDP/DNA CLPNs were prepared with different concentrations of cationic lipid (DOTAP) to polymer (PLGA) ratio (5, 10, 15, 20 and 25\%), named CDDP/DNA CLPNs 1 (5\%), CDDP/DNA CLPNs 2 (10\%), CDDP/DNA CLPNs 3 (15\%), CDDP/DNA CLPNs 4 (20\%), CDDP/DNA CLPNs 5 (25\%).

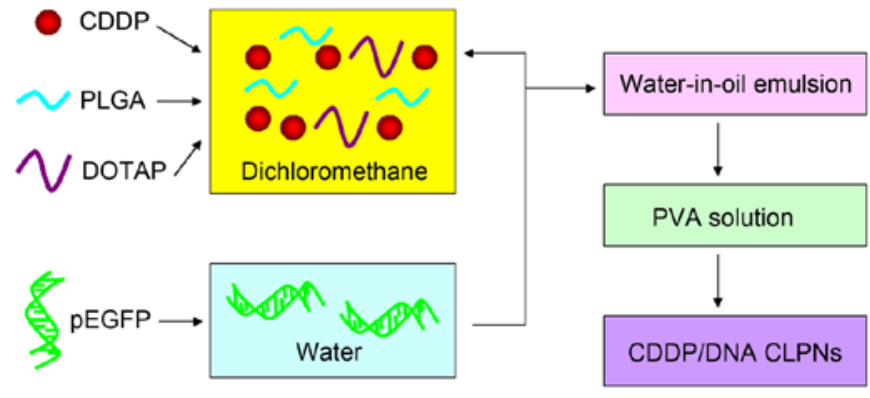

Figure 1. Scheme of the preparation of CDDP/DNA CLPNs. CDDP, cisplatin; CLPNs, cationic lipid-polymer hybrid nanoparticles.

CDDP CLPNs were prepared by the same method without the use of pEGFP.

Blank CLPNs were prepared by the same method without the use of pEGFP and CDDP.

Preparation of CDDP PNPs. CDDP PNPs were prepared by the emulsion solvent evaporation method (22). Briefly, CDDP $(0.1 \mathrm{~g})$, PLGA $(0.3 \mathrm{~g})$ were dissolved in dichloromethane $(10 \mathrm{ml})$ to form the organic phase. The organic phase was added drop-wise into $1 \% \mathrm{w} / \mathrm{v}$ PVA aqueous solution being stirred at $400 \mathrm{rpm}$ at room temperature until the evaporation of dichloromethane was complete. Then the resulting suspension was washed three times by repeating the centrifugation step and freeze-dried at $-20^{\circ} \mathrm{C}$ to get the final formulated CDDP PNPs.

Blank PNPs were prepared by the same method without the use of CDDP.

Preparation of DNA LIPO. For the comparison of the gene transfection efficacy of CDDP/DNA CLPNs, DNA LIPO complexes were prepared by the following method (12): DNA and Lipofectamine ${ }^{\circledR} 2000$ (1:2 w/w) was mixed for $30 \mathrm{sec}$ using a vortex mixer. Then, the DNA LIPO was obtained by incubating the mixture for $30 \mathrm{~min}$ and freeze-dried at $-20^{\circ} \mathrm{C}$.

\section{Characterization}

Morphology. The morphology of CDDP/DNA CLPNs, and CDDP PNPs was examined by transmission electron microscopy (TEM) (JEM-200CX; JEOL Co., Ltd., Tokyo, Japan) (23). Samples were stained at room temperature with freshly prepared and sterile-filtered $1 \%(\mathrm{w} / \mathrm{v})$ phosphotungstic acid aqueous solution. The samples were then placed in a carbon-coated copper grid and air-dried prior to imaging (24).

Size and surface charge. The mean particle diameter, size distribution (polydispersity index), and surface charge ( $\zeta$ potential) of CDDP/DNA CLPNs and other nanoparticles were determined by dynamic light scattering (DLS) using a Zetasizer Nano ZS (Malvern Instruments, Malvern, UK) (25).

DNA-binding efficiency $(D E)$. The DE of CDDP/DNA CLPNs was determined by the PicoGreen fluorometry method (26). Briefly, free pEGFP was isolated from the CDDP/DNA CLPNs by centrifugation at 15,000 rpm and $4^{\circ} \mathrm{C}$ for $30 \mathrm{~min}$, the supernatants were collected, and the concentration of pEGFP was assessed by a fluorescence spectrophotometer $(\mathrm{Ex} / \mathrm{Em}=480 / 520 \mathrm{~nm})($ Hitachi F-2500; 
Hitachi High-Technologies Corp., Tokyo, Japan). The amount of DNA loaded in the CDDP/DNA CLPNs was calculated as:

$$
\text { DE }(\%)=\frac{\begin{array}{c}
\text { weight of DNA } \\
\text { totally added }
\end{array}-\begin{array}{c}
\text { weight of free } \\
\text { DNA tested }
\end{array}}{\text { weight of DNA totally added }} \times 100
$$

$C D D P$ encapsulation efficiency (CE). The $\mathrm{CE}$ in the CDDP/DNA CLPNs and CDDP PNPs was determined by inductively coupled plasma-optical emission spectrometry (ICP-OES) (27). The detection was carried out at $\lambda=265.9 \mathrm{~nm}$. The amount of CDDP was calculated as:

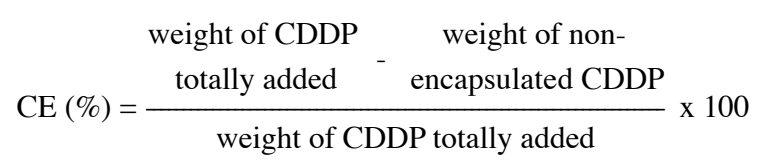

Plasma stability. The plasma stability of the CDDP/DNA CLPNs and other nanoparticles was determined in the presence of $50 \%$ plasma (v/v) $(28,29)$. The formulations were incubated in phosphate-buffered saline (PBS) solution containing 50\% FBS at $37^{\circ} \mathrm{C}$ for $24 \mathrm{~h}$, separately. At scheduled times $(0,2,4,8$, 12 and $24 \mathrm{~h}$ ), $1 \mathrm{ml}$ of each sample was diluted with $2 \mathrm{ml}$ THF and the mixture was bath-sonicated for $5 \mathrm{~min}$, followed by centrifugation at 10,000 rpm for $5 \mathrm{~min}$. The variation trends of the size, CE, and DE were calculated by the method indicated above.

Cells. Human RMS cell line, RD-4 cells, were obtained from the American Type Culture Collection (Manassas, VA, USA) and cultured at $37^{\circ} \mathrm{C}$ and $5 \% \mathrm{CO}_{2}$ in DMEM supplemented with $10 \%$ FBS and $1 \%$ penicillin $(5,000 \mathrm{U} / \mathrm{ml})$-streptomycin $(5,000 \mathrm{U} / \mathrm{ml})$.

In vitro cytotoxicity. The in vitro cytotoxicity of CDDP/DNA CLPNs and other nanoparticles was evaluated on RD-4 cells by MTT assay (30). Cells cultured in the above section were seeded in a 96-well plate at a seeding density of $10^{4}$ cells/well. The culture medium was then replaced with various doses of CDDP/DNA CLPNs and other nanoparticles. Culture medium was used as a blank control. After $48 \mathrm{~h}$ of incubation, the media were replaced with $90 \mu \mathrm{l}$ of free-serum medium and $10 \mu \mathrm{l}$ of MTT solution ( $5 \mathrm{mg} / \mathrm{ml}$ in sterile PBS). After a 4-h incubation at $37^{\circ} \mathrm{C}$, the MTT solution in the wells was replaced with $100 \mu 1$ dimethylsulfoxide (DMSO). The absorption at $570 \mathrm{~nm}$ (OD570) was measured on a spectrophotometer. The cell viability was converted and expressed as the percentage of the control, and the $\mathrm{IC}_{50}$ values were calculated accordingly.

In vitro gene transfection. The in vitro transfection efficacy of CDDP/DNA CLPNs and DNA LIPO was evaluated on RD-4 cells, using CDDP CLPNs and blank CLPNs as control (24). Cells cultured in the above section were seeded in a 96-well plate at a seeding density of $10^{4}$ cells/well, $24 \mathrm{~h}$ prior to transfection. The media were replaced with $200 \mu \mathrm{l}$ serum-free media containing CDDP/DNA CLPNs and other nanoparticles at $37^{\circ} \mathrm{C}$. Naked DNA solution was used as a negative control. DNA LIPO was used as a positive control. The original incubation medium was replaced with $1 \mathrm{ml}$ of complete medium after incubation for $4 \mathrm{~h}$ at $37^{\circ} \mathrm{C}$ in a $5 \% \mathrm{CO}_{2}$ incubator, and then cells were incubated sequentially until $48 \mathrm{~h}$ post-transfection.

Flow cytometry was carried out for the quantitation of the cells which were successfully transfected. At the end of the incubation, cells were washed once with $1 \mathrm{ml}$ of PBS and were detached with trypsin/EDTA. Then the cells were centrifuged at $1,500 \mathrm{rpm}$, at $4^{\circ} \mathrm{C}$ for $5 \mathrm{~min}$, the supernatant was discarded, and the cells were washed once with $1 \mathrm{ml}$ of PBS, centrifuged again $\left(1,500 \mathrm{rpm}, 4^{\circ} \mathrm{C}\right.$ for $\left.5 \mathrm{~min}\right)$, the supernatant was discarded, and the cells were re-suspended in $300 \mu$ of PBS and directly introduced to a BD FACSCalibur flow cytometer (Becton, Dickinson and Co., Franklin Lakes, NJ, USA).

Animals. BALB/c nude mice (5-6 week-old, 18-22 g) were purchased from the Shanghai Slack Laboratory Animal Co., Ltd. (Shanghai, China) and were maintained under specific pathogen-free conditions. Ethics approval was received from the Medical Ethics Committee of Kunming Medical University (no. KMMU20160316-1).

In vivo antitumor efficacy. The in vivo antitumor efficacy of CDDP/DNA CLPNs and other nanoparticles was investigated in RD-4 tumor-bearing BALB/c nude mouse models, which were developed by injection of RD-4 cells in the right armpit of BALB/c mice (31). When tumor volume reached $\sim 50 \mathrm{~mm}^{3}$, transplanted mice were randomly divided into seven groups (8 mice/group) separately. Formulations for the seven groups were as follows: group 1, 0.9\% saline (control group); group 2, CDDP solution $(8 \mathrm{mg} / \mathrm{kg})$; group 3, blank PNPs; group 4, CDDP PNPs (4 mg/kg); group 5, blank CLPNs; group 6, CDDP CLPNs (4 mg/kg); group 7, CDDP/DNA CLPNs $(4 \mathrm{mg} / \mathrm{kg})$. The mice of each group were given the above formulations by tail vein injection once every 3 days. Three weeks later, all the mice were sacrificed by cervical dislocation and the tumor tissue samples were taken out. Tumor volume of each mouse was measured with a digital caliper every 3 days, and was calculated according to the equation:

$$
\mathrm{V}\left(\mathrm{mm}^{3}\right)=\frac{(\text { major axis }) \times(\text { minor axis })^{2}}{2}
$$

The antitumor efficacy of each formulation was evaluated by tumor inhibition rate (TIR), which was calculated by measuring the tumor weight (TW) using the following formula:

$$
\operatorname{TIR}(\%)=\frac{\begin{array}{c}
\mathrm{TW} \text { of } \\
\text { control group }
\end{array}-\begin{array}{c}
\mathrm{TW} \text { of } \\
\text { treated group }
\end{array}}{\mathrm{TW} \text { of control group }}
$$

In vivo gene delivery. The $\mathrm{RD}-4$ tumor-bearing $\mathrm{BALB} / \mathrm{c}$ nude mouse model was designed by the method indicated in the above section. Then, six groups of tumor-bearing mice received a 300- $\mu 1$ intravenous injection: group $1,0.9 \%$ saline (control group); group 2, naked DNA solution; group 3, DNA LIPO; group 4, blank CLPNs; group 5, CDDP CLPNs; group 6, CDDP/DNA CLPNs, respectively. After $48 \mathrm{~h}$, mice were sacrificed by cervical dislocation, and their tumor tissues were taken and washed with cold saline twice. Tissues were homogenized in lysis buffer $(0.05 \%$ Triton X-100, 2 mM EDTA, 
Table I. Optimization and characterization of nanoparticles.

\begin{tabular}{|c|c|c|c|c|c|}
\hline Nanoparticles & $\begin{array}{c}\text { Particle } \\
\text { diameter }(\mathrm{nm})\end{array}$ & $\begin{array}{l}\text { Polydispersity } \\
\text { index }\end{array}$ & $\begin{array}{c}\text { Surface } \\
\text { charge }(\mathrm{mV})\end{array}$ & $\mathrm{DE}(\%)$ & $\mathrm{CE}(\%)$ \\
\hline DNA LIPO & $139.4 \pm 4.8$ & $0.10 \pm 0.015$ & $+43.1 \pm 4.7$ & $91.5 \pm 3.9$ & N/A \\
\hline Blank PNPs & $78.3 \pm 2.9$ & $0.08 \pm 0.009$ & $-26.3 \pm 2.8$ & N/A & N/A \\
\hline CDDP PNPs & $82.1 \pm 3.2$ & $0.09 \pm 0.010$ & $-28.9 \pm 3.2$ & N/A & $83.6 \pm 2.4$ \\
\hline Blank CLPNs & $118.3 \pm 3.8$ & $0.12 \pm 0.013$ & $+41.6 \pm 2.5$ & N/A & N/A \\
\hline CDDP CLPNs & $117.6 \pm 4.4$ & $0.16 \pm 0.012$ & $+39.8 \pm 3.3$ & N/A & $85.3 \pm 2.8$ \\
\hline CDDP/DNA CLPNs 1 & $335.2 \pm 36.8$ & $0.67 \pm 0.094$ & $+6.6 \pm 1.4$ & $35.7 \pm 7.4$ & $17.5 \pm 5.3$ \\
\hline CDDP/DNA CLPNs 2 & $243.1 \pm 21.9$ & $0.36 \pm 0.062$ & $+12.8 \pm 2.7$ & $53.8 \pm 8.9$ & $63.8 \pm 6.5$ \\
\hline CDDP/DNA CLPNs 3 & $137.6 \pm 3.3$ & $0.10 \pm 0.008$ & $+25.4 \pm 3.5$ & $90.7 \pm 3.1$ & $82.1 \pm 2.7$ \\
\hline CDDP/DNA CLPNs 4 & $133.6 \pm 3.7$ & $0.12 \pm 0.010$ & $+31.1 \pm 4.4$ & $88.6 \pm 4.2$ & $62.5 \pm 4.9$ \\
\hline CDDP/DNA CLPNs 5 & $141.2 \pm 4.5$ & $0.13 \pm 0.014$ & $+36.9 \pm 3.8$ & $89.3 \pm 6.1$ & $51.6 \pm 8.3$ \\
\hline
\end{tabular}

DE, DNA-binding efficiency; CE, CDDP encapsulation efficiency; DNA LIPO, DNA-loaded Lipofectamine ${ }^{\circledR}$ 2000; PNPs, polymeric nanoparticles; CDDP, cisplatin; CLPNs, cationic lipid-polymer hybrid nanoparticles.

$0.1 \mathrm{M}$ Tris- $\mathrm{HCl}, \mathrm{pH}$ 7.8). After several cycles of freezing and thawing, the homogenates were centrifuged at $10,000 \mathrm{rpm}$ for 5 min to obtain the cells. Cells were cultured by the method of 'In vitro cytotoxicity' section.

For qualitative analysis, fluorescent cells were observed using an inverted fluorescence microscope (Olympus ZX71; Olympus Corp., Tokyo, Japan) and the picture was captured. The transfection efficiency of the fluorescent cells was quantified using the flow cytometry method described in the 'In vitro transfection' section.

Statistical analysis. The data are presented as means \pm standard deviation (SD). Statistical analysis was performed by a one-tailed Student's t-test in Excel. Statistical significance was established at the $5 \%$ level $(\mathrm{P}<0.05)$ for all statistical analyses.

\section{Results}

Optimization of CDDP/DNA CLPNs. In order to optimize cationic lipid (DOTAP) to polymer (PLGA) ratio, CDDP/DNA CLPNs with different concentrations of lipids were prepared using different ratios of cationic lipid to polymer: 5, 10, 15, 20 and $25 \%$. The influence of the lipid to the polymer ratio was determined in terms of particle size, polydispersity index, surface charge, DE and CE (Table I).

It was observed that the particle diameters of CDDP/DNA CLPNs 1, CDDP/DNA CLPNs 2, CDDP/DNA CLPNs 3, CDDP/DNA CLPNs 4, and CDDP/DNA CLPNs 5 were 335.2, 243.1, 137.6, 133.6 and $141.2 \mathrm{~nm}$, respectively. The polydispersity index of CDDP/DNA CLPNs 1 (0.67) and CDDP/DNA CLPNs 2 (0.36) were significantly higher than other formulations, suggesting that the ratios of 5 and $10 \%$ were not suitable. The obviously lower DE and CE of CDDP/DNA CLPNs 1 and CDDP/DNA CLPNs 2 also confirmed this conclusion. The CE of CDDP/DNA CLPNs 4 (62.5) and CDDP/DNA CLPNs 5 (51.6) were significantly lower than CDDP/DNA CLPNs 3 (82.1) $(\mathrm{P}<0.05)$. So the optimized lipid (DOTAP) to polymer (PLGA) ratio was $15 \%$ (CDDP/DNA CLPNs 3).

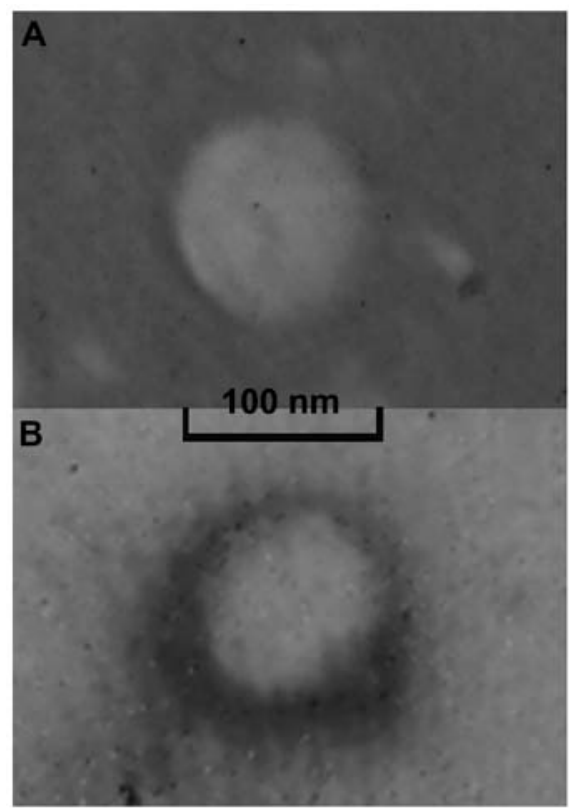

Figure 2. TEM images of (A) CDDP/DNA CLPNs and (B) CDDP PNPs TEM, transmission electron microscopy; CDDP, cisplatin; CLPNs, cationic lipid-polymer hybrid nanoparticles; PNPs, polymeric nanoparticles.

\section{Characterization}

Morphology. The overall morphology of the CDDP/DNA CLPNs was a clear core-shell type spherical nanoparticle (Fig. 2A). The core-shell structure was clearly visible with a white core and the shell is grey. In comparison, the morphology of CDDP PNPs was white spherical particle without outer layer (Fig. 2B). The size scales in the images show that the size of CDDP/DNA CLPNs was slightly larger than $100 \mathrm{~nm}$, while the diameter of CDDP PNPs was smaller than $100 \mathrm{~nm}$.

Size, surface charge, $D E$ and $C E$. The size of CDDP/DNA CLPNs, CDDP CLPNs, blank CLPNs, CDDP PNPs, and blank PNPs was 137.6 $\pm 3.3,117.6 \pm 4.4,118.3 \pm 3.8$, $82.1 \pm 3.2$ and $78.3 \pm 2.9 \mathrm{~nm}$, respectively. The size of CDDP 

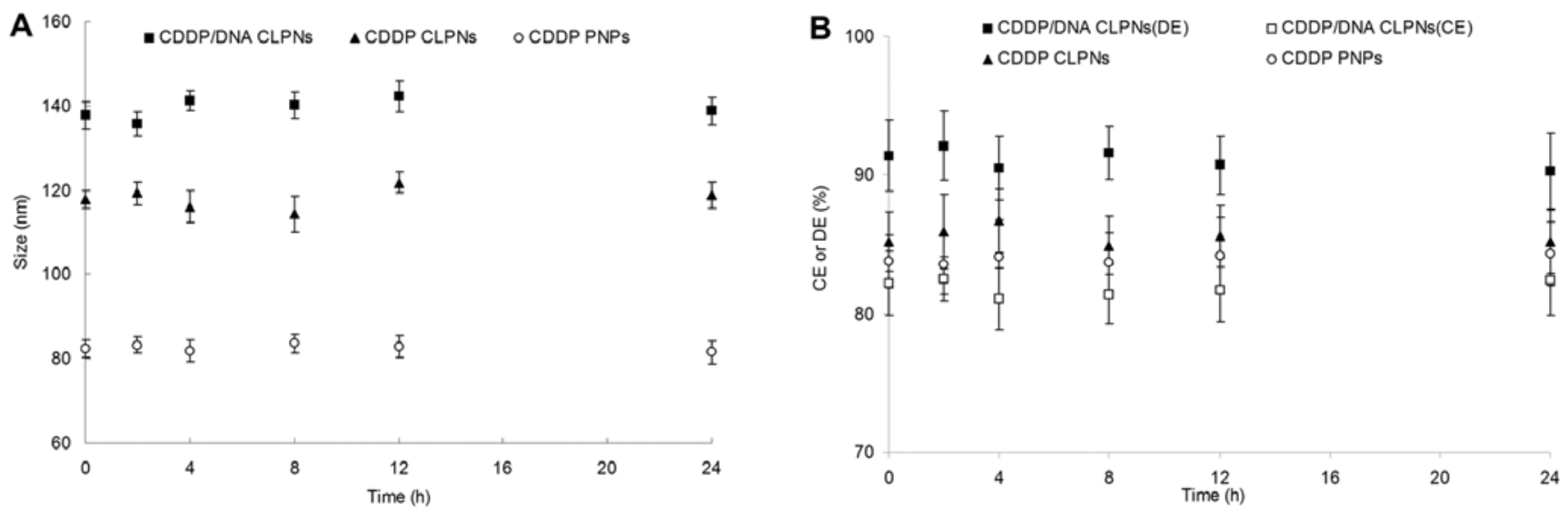

Figure 3. Changes in (A) size, (B) drug- and gene-loading efficiency of different particles in the presence of serum.

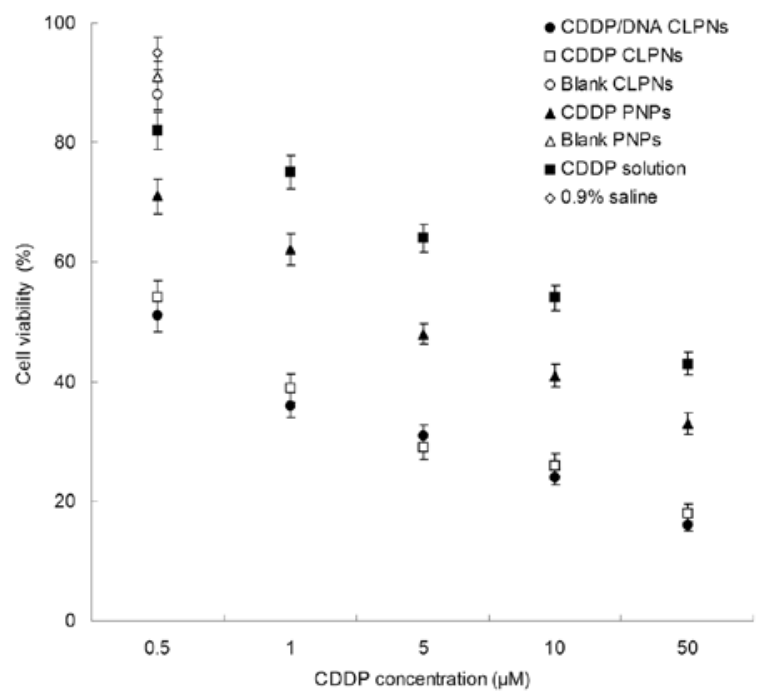

Figure 4. Cell viability of various concentrations of nanoparticles for $48 \mathrm{~h}$ in RMS cell lines. RMS, rhabdomyosarcoma.

Table II. IC $_{50}$ values of samples.

\begin{tabular}{lcccc}
\hline Samples & $\begin{array}{c}\text { CDDP/DNA } \\
\text { CLPNs }\end{array}$ & $\begin{array}{c}\text { CDDP } \\
\text { CLPNs }\end{array}$ & $\begin{array}{c}\text { CDDP } \\
\text { PNPs }\end{array}$ & $\begin{array}{c}\text { CDDP } \\
\text { solution }\end{array}$ \\
\hline $\begin{array}{lccc}\mathrm{IC}_{50} \text { of } \\
\mathrm{CDDP}(\mu \mathrm{M})\end{array}$ & $0.54 \pm 0.06$ & $0.58 \pm 0.07$ & $4.12 \pm 0.36$ & $20.36 \pm 3.85$ \\
\hline
\end{tabular}

CDDP, cisplatin; CLPNs, cationic lipid-polymer hybrid nanoparticles; PNPs, polymeric nanoparticles.

CLPNs and blank CLPNs, CDDP PNP and blank PNPs had no obvious difference $(\mathrm{P}>0.05)$. The size of CDDP CLPNs was larger than CDDP PNPs $(\mathrm{P}<0.05)$. The size of CDDP/DNA CLPNs was the largest.

The surface charge of CDDP/DNA CLPNs, CDDP CLPNs, and CDDP PNPs was $+25.4 \pm 3.5,+39.8 \pm 3.3$ and $-28.9 \pm 3.2 \mathrm{mV}$. CDDP PNPs have negative $\zeta$ potential. In contrast, CLPNs have positive charges. The surface charge of CDDP/DNA CLPNs was lower than CDDP CLPNs.

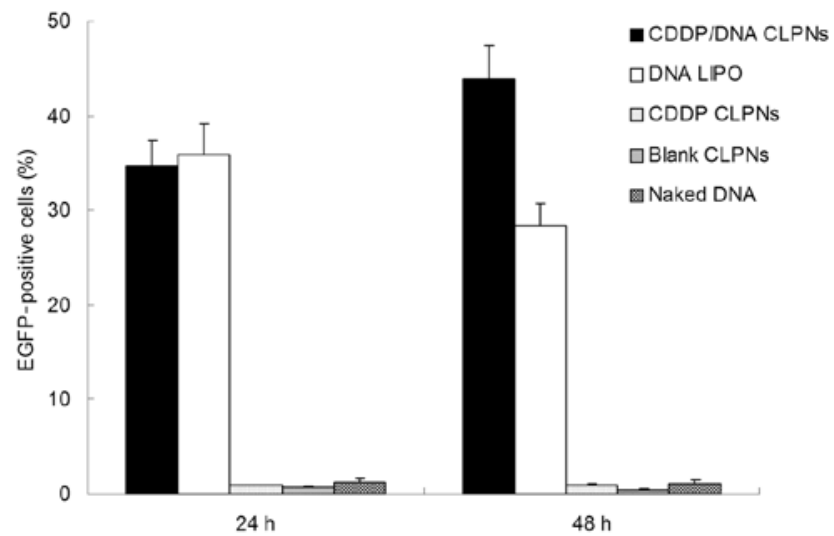

Figure 5. In vitro transfection efficacy of CDDP/DNA CLPNs and DNA LIPO evaluated on RD-4 cells. CDDP, cisplatin; CLPNs, cationic lipid-polymer hybrid nanoparticles; DNA LIPO, DNA-loaded Lipofectamine ${ }^{\circledR} 2000$.

The DE of CDDP/DNA CLPNs and DNA LIPO was $90.7 \pm 3.1$ and $91.5 \pm 3.9 \%$, respectively. The CE of CDDP/DNA CLPNs, CDDP CLPNs, and CDDP PNPs was 82.1 \pm 2.7 , $85.3 \pm 2.8$ and $83.6 \pm 2.4$, respectively. These results illustrated that the loading capacity of CDDP and binding ability of DNA was outstanding for all the drug-loaded nanoparticles prepared in this study.

Plasma stability. Fig. 3 illustrated the changes in size, drug and gene-loading efficiency of different particles in the presence of serum. The particle diameter, CE or DE had no significant variation up to $24 \mathrm{~h}$. Due to these results, CDDP/DNA CLPNs, and CDDP PNPs were considered very stable after intravenous administration.

In vitro cytotoxicity. As shown in Fig. 4, treatment with various concentrations of CDDP-loaded particles for $48 \mathrm{~h}$ caused various viability reductions in RMS cell lines. $\mathrm{IC}_{50}$ of CDDP/DNA CLPNs $(0.54 \pm 0.06 \mu \mathrm{M})$ and CDDP CLPNs $(0.58 \pm 0.07 \mu \mathrm{M})$ showed no significant difference in RD-4 cells (Table II). The cytotoxicity of CDDP/DNA CLPNs was significantly higher $\left(\mathrm{IC}_{50}\right.$ was 8 times dose advantage) than CDDP PNPs $(4.12 \pm 0.36 \mu \mathrm{M}) . \mathrm{IC}_{50}$ of CDDP solution was $20.36 \pm 3.85 \mu \mathrm{M}$.

In vitro gene transfection. Fig. 5 exhibits the in vitro transfection efficacy of CDDP/DNA CLPNs and DNA LIPO 
Table III. TW and TIR of samples.

\begin{tabular}{lccccc}
\hline Samples & CDDP/DNA CLPNs & CDDP CLPNs & CDDP PNPs & CDDP solution & $0.9 \%$ Saline \\
\hline TW $(\mathrm{g})$ & $0.12 \pm 0.02$ & $0.11 \pm 0.02$ & $0.61 \pm 0.09$ & $0.95 \pm 0.12$ \\
TIR $(\%)$ & 90 & 91 & 52 & 25 & $1.26 \pm 0.23$ \\
& & & 100 & 25 \\
\hline
\end{tabular}

TW, tumor weight; TIR, tumor inhibition rate; CDDP, cisplatin; CLPNs, cationic lipid-polymer hybrid nanoparticles; PNPs, polymeric nanoparticles.

evaluated on RD-4 cells. Gene transfection efficiency was measured by analyzing the expression of the EGFP by flow cytometry. When delivering genes into RD-4 cells for $24 \mathrm{~h}$, CDDP/DNA CLPNs showed the same transfection efficiency as DNA LIPO ( $\mathrm{P}>0.05)$. However, after 48 -h post-transfection, the CDDP/DNA CLPNs was more efficient than the DNA LIPO $(\mathrm{P}<0.05)$. Naked DNA solution group got no obvious gene expression, indicating that DNA cannot successfully transfect the cancer cells without carriers. CDDP CLPN and blank CLPN groups showed no gene expression in RD-4 cells, ruling out the fluorescent cells caused by the CLPNs and CDDP.

In vivo antitumor. Fig. 6 shows the in vivo antitumor efficacy of CDDP/DNA CLPNs and other nanoparticles was investigated in RD-4 tumor-bearing BALB/c nude mouse models. CDDP/DNA CLPNs, CDDP CLPNs, CDDP PNPs and CDDP solutions showed significant tumor regression in tumor-bearing mice, with a reduction in tumor volume. Mice in the other three groups shared similar tumor-growth pattern. At 21 days of administration, the TW and TIR of tumor-bearing mice are given in Table III. CDDP/DNA CLPN and CDDP CLPN groups exhibited the highest TIR (90 and 91\%), followed by CDDP PNPs (52\%) and CDDP solution (25\%).

In vivo gene delivery. Fig. 7 illustrates the images of the fluorescent cells after $48 \mathrm{~h}$ of administration. The highest transfection efficiency appeared in the pictures is CDDP/DNA CLPNs group. The image of DNA LIPO group came in the second place. The naked DNA solution group has slightly weaker fluorescence. Other groups do not have any fluorescence according to the images. The transfection efficiency of the fluorescent cells was quantified using a flow cytometry method (Fig. 8). Substantially higher transfection efficiency was observed in CDDP/DNA CLPNs than DNA LIPO group at both $24-$ and 48 -h post-administration $(\mathrm{P}<0.05)$. One explanation may be that as an in vitro transfection vector, Lipofectamine ${ }^{\circledR} 2000$ transfection reagent was not so stable and might have some toxicity in vivo. The highest in vivo gene transfection efficiency of CDDP/DNA CLPNs was detected after $48 \mathrm{~h}$, showing the stability and controlled release of the CLPNs.

\section{Discussion}

The aim of the present study was to develop nanoparticles for co-delivery of CDDP and DNA. The influence of cationic lipid concentration on the characteristics of CLPNs was evaluated. In order to optimize cationic lipid (DOTAP) to

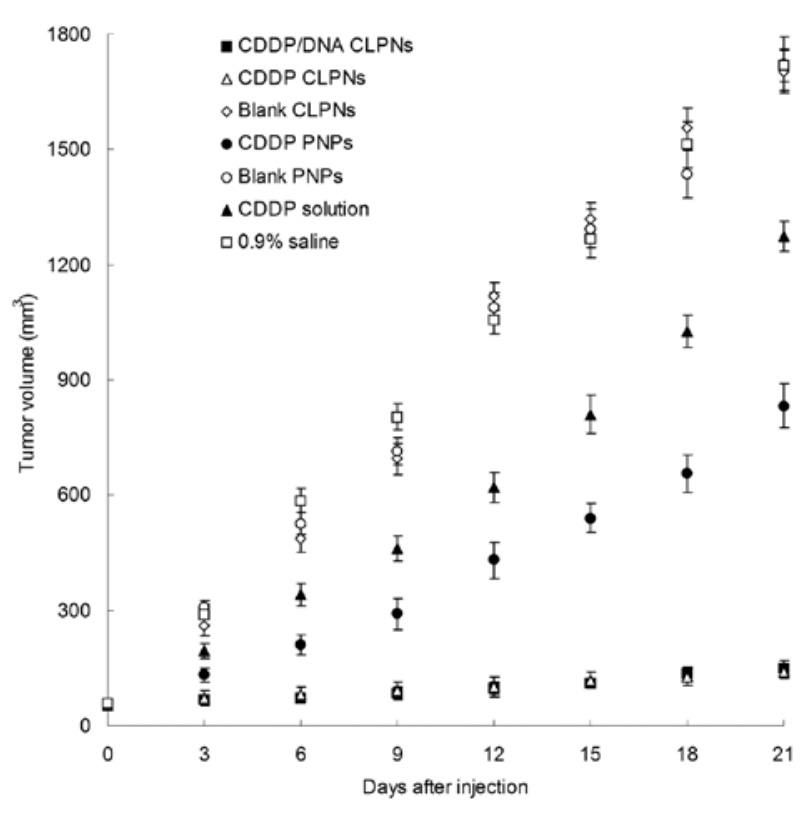

Figure 6. In vivo antitumor efficacy of CDDP/DNA CLPNs and other nanoparticles in RD-4 tumor-bearing BALB/c nude mouse models. CDDP, cisplatin; CLPNs, cationic lipid-polymer hybrid nanoparticles.

polymer (PLGA) ratio, CDDP/DNA CLPNs with different concentrations of lipids were prepared using different ratios of cationic lipid to polymer: 5, 10, 15, 20 and 25\%. The influence of the lipid to polymer ratio was determined in terms of particle size, polydispersity index, surface charge, DE and CE (Table I). It was observed that the particle diameters of CDDP/DNA CLPNs 1, CDDP/DNA CLPNs 2, CDDP/DNA CLPNs 3, CDDP/DNA CLPNs 4, and CDDP/DNA CLPNs 5 were 335.2, 243.1, 137.6, 133.6 and $141.2 \mathrm{~nm}$, respectively. It was also found that there was a decrease in particle size as the lipid concentrations increased. The concentration of cationic lipids could play a significant role in controlling the size of CDDP/DNA CLPNs, possibly reducing the coalescence of particles (32). The polydispersity index of CDDP/DNA CLPNs 1 (0.67) and CDDP/DNA CLPNs 2 (0.36) was significantly higher than other formulations, suggesting that the ratios of 5 and $10 \%$ were not suitable. The obviously lower DE and CE of CDDP/DNA CLPNs 1 and CDDP/DNA CLPNs 2 also confirmed this conclusion. The CE of CDDP/DNA CLPNs 4 (62.5) and CDDP/DNA CLPNs 5 (51.6) were significantly lower than CDDP/DNA CLPNs 3 (82.1) $(\mathrm{P}<0.05)$. So the optimized lipid (DOTAP) to polymer (PLGA) ratio was $15 \%$.

The core-shell structure of CDDP/DNA CLPNs was clearly visible with a white core and the shell is grey (Fig. 2). 

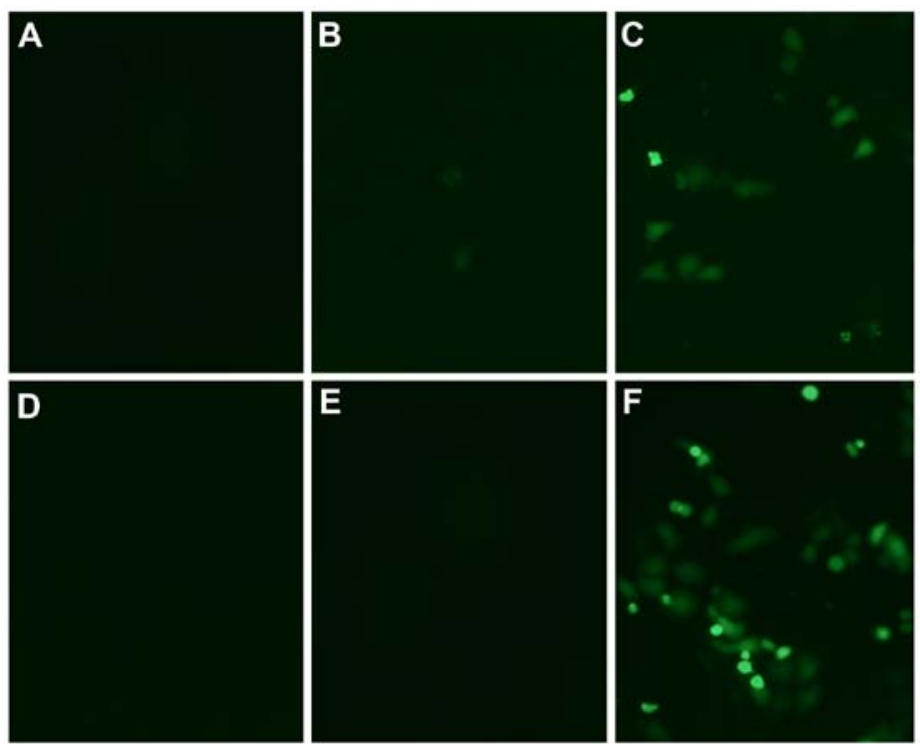

Figure 7. Images of the fluorescent cells for (A) $0.9 \%$ saline (control group), (B) naked DNA solution, (C) DNA LIPO, (D) blank CLPNs, (E) CDDP CLPNs, and (F) CDDP/DNA CLPNs in the evaluation of in vivo gene delivery (48 hafter administration). DNA LIPO, DNA-loaded Lipofectamine ${ }^{\circledR} 2000$; CLPNs, cationic lipid-polymer hybrid nanoparticles; CDDP, cisplatin.

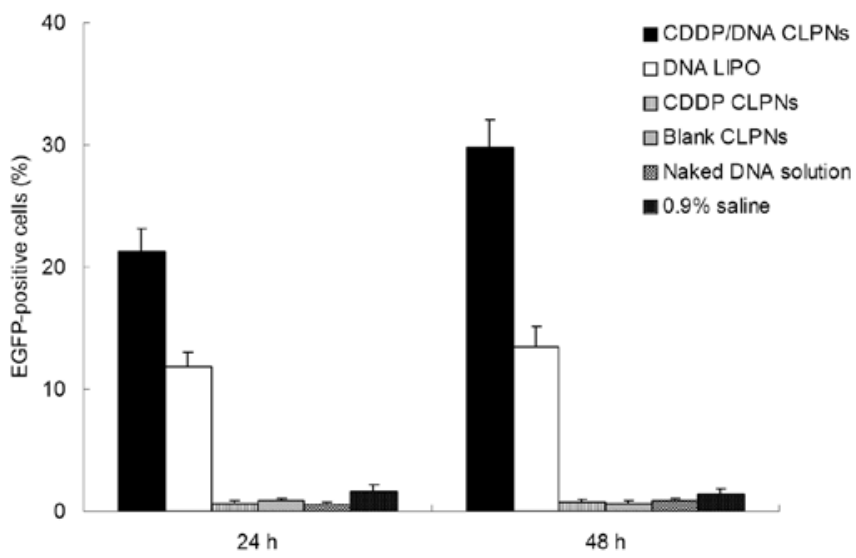

Figure 8. In vitro transfection efficacy of CDDP/DNA CLPNs and DNA LIPO evaluated in RD-4 tumor-bearing BALB/c nude mouse models. CDDP, cisplatin; CLPNs, cationic lipid-polymer hybrid nanoparticles; DNA LIPO, DNA-loaded Lipofectamine ${ }^{\circledR} 2000$.

In comparison, the morphology of CDDP PNPs was white spherical particles without outer layer. The TEM image illustrates the self-assembly process of the lipid-polymer core-shell structure by forming a lipid layer on the surface of the inner polymer core $(33,34)$. The size scales in the images show that the size of CDDP/DNA CLPNs was slightly larger than $100 \mathrm{~nm}$, while the diameter of CDDP PNPs was smaller than $100 \mathrm{~nm}$.

The size of CDDP CLPNs and blank CLPNs, CDDP PNP and blank PNPs had no obvious difference $(\mathrm{P}>0.05)$. This means that the loading of CDDP did not change the size of the particles. The size of CDDP CLPNs was larger than CDDP PNPs $(\mathrm{P}<0.05)$. This could be explained by the lipid shell on the polymer core enlarged the size of the particles. The size of CDDP/DNA CLPNs was the largest; indicating that the DNA could attribute to the increase of the particle diameter. Surface charge is an important indication of the stability of a colloidal system in a particular medium (35). The surface charge of CDDP/DNA CLPNs, CDDP CLPNs, and CDDP PNPs was $+25.4 \pm 3.5,+39.8 \pm 3.3$ and $-28.9 \pm 3.2 \mathrm{mV}$. For CDDP PNPs, negatively charged PLGA gave the PNPs negative $\zeta$ potential. In contrast, inclusion of cationic lipids changed the surface charges of the particles; CLPNs have positive charges. The surface charge of CDDP/DNA CLPNs was lower than CDDP CLPNs, suggesting that the combination of anionic DNA decreased the charge of the CLPNs. The DE of CDDP/DNA CLPNs and DNA LIPO was $~ 90 \%$. The CE of CDDP/DNA CLPNs, CDDP CLPNs, and CDDP PNPs was $>80 \%$. These results illustrated that the loading capacity of CDDP and binding ability of DNA was outstanding for all the drug-loaded nanoparticles prepared in this study.

The plasma stability of the CDDP/DNA CLPNs and other nanoparticles was determined in the presence of plasma. The particle diameter, $\mathrm{CE}$ or DE had no significant variation up to $24 \mathrm{~h}$ (Fig. 3). This stability would be advantageous when the complexes are administered in vivo. One of the major concerns for cationic non-viral vectors is the disassociation of DNA from the vectors due to electrostatic interaction between the vectors and the plasma composition such as proteins (29). The dissociated DNA would be quickly degraded by enzymolysis. Thus, the stability of the cationic non-viral vectors in human plasma is one of the most essential properties. Also the stability in the plasma is required for the drug-loaded nanoparticles. These would increase the stability of the loaded drug and DNA during the circulation after intravenous administration and enhance the targeting proportion of the vector to the target organ, and thus would be favorable to improve the therapeutic effects.

In vitro cytotoxicity of CDDP/DNA CLPNs was evaluated against RD-4 cells, and was compared with the cytotoxicity induced by free-CDDP solution, blank nanoparticles, and $0.9 \%$ saline solution. As showed in Fig. 4, treatment with various concentrations of CDDP-loaded particles for $48 \mathrm{~h}$ caused various viability reductions in RMS cell lines. $\mathrm{IC}_{50}$ 
of CDDP/DNA CLPNs $(0.54 \pm 0.06 \mu \mathrm{M})$ and CDDP CLPNs $(0.58 \pm 0.07 \mu \mathrm{M})$ showed no significant difference in RD-4 cells, indicating that DNA does not affect the ability of the CDDP loaded in the CLPNs (Table I) $(\mathrm{P}>0.05)$. The cytotoxicity of CDDP/DNA CLPNs was significantly higher $\left(\mathrm{IC}_{50}\right.$ was 8 times dose advantage) than CDDP PNPs $(4.12 \pm 0.36 \mu \mathrm{M})$. This result may suggest that the in vitro tumor cell inhibition of drug-loaded CLPNs was more efficient than drug-loaded PNPs (P<0.05). $\mathrm{IC}_{50}$ of CDDP solution was $20.36 \pm 3.85 \mu \mathrm{M}$. These results indicate that CDDP/DNA CLPNs exhibited superior cytotoxicity to RD-4 cells as evidenced by its significantly decreased $\mathrm{CDDP}_{\mathrm{IC}} \mathrm{C}_{50}$ values.

In vitro transfection efficacy of CDDP/DNA CLPNs and DNA LIPO was evaluated on RD-4 cells, using CDDP CLPNs and blank CLPNs as control. Gene transfection efficiency was measured by analyzing the expression of the EGFP by flow cytometry. When delivering genes into RD-4 cells for $24 \mathrm{~h}$, CDDP/DNA CLPNs showed the same transfection efficiency as DNA LIPO ( $\mathrm{P}>0.05)$. However, after 48 -h post-transfection, the CDDP/DNA CLPNs were more efficient than the DNA LIPO $(\mathrm{P}<0.05)$. This could be explained by the polymer core and the lipid shell controlling the release of the DNA thus gaing the long-lasting efficiency (36). Naked DNA solution group had no obvious gene expression; indicating that DNA cannot successfully transfect the cancer cells without carriers. CDDP CLPNs and blank CLPN groups showed no gene expression in RD-4 cells, ruling out the fluorescent cells caused by the CLPNs and CDDP. The results illustrated that CLPNs are able to condense DNA efficiently, deliver DNA into the cells by ionic interactions with the cell membrane and release DNA into the cytoplasm from endocytic vesicles (37). Better transfection efficiency than commercial Lipofectamine 2000 and DNA complex indicates that the CLPNs can be a good transfection reagent (38).

In vivo antitumor efficacy of CDDP/DNA CLPNs and other nanoparticles was investigated in RD-4 tumor-bearing BALB/c nude mouse models (Fig. 5). CDDP/DNA CLPNs, CDDP CLPNs, CDDP PNPs and CDDP solution showed significant tumor regression in tumor-bearing mice, with a reduction of tumor volume. Mice in the other three groups shared similar tumor-growth pattern, suggesting that blank carriers were not capable of inhibiting tumor growth (19). At 21 days of administration, the TW and TIR of tumor-bearing mice are shown in Table III. CDDP/DNA CLPN and CDDP CLPN groups exhibited the highest TIR (90 and 91\%), followed by CDDP PNPs (52\%) and CDDP solution (25\%). Fig. 7 illustrates the images of the fluorescent cells. The highest transfection efficiency appearing in the images is in CDDP/DNA CLPN group. The image of DNA LIPO group comes in the second place. The naked DNA solution group has slightly weaker fluorescence. Other groups have no fluorescence according to the images. The transfection efficiency of the fluorescent cells was quantified using a flow cytometry method (Fig. 8). Significantly higher transfection efficiency was observed in CDDP/DNA CLPNs than DNA LIPO group at both $24-$ and $48-\mathrm{h}$ post-administration $(\mathrm{P}<0.05)$. One explanation may be that as an in vitro transfection vector, Lipofectamine ${ }^{\circledR} 2000$ Transfection Reagent was not so stable and might have some toxicity in vivo. The highest in vivo gene transfection efficiency of CDDP/DNA CLPNs was detected after $48 \mathrm{~h}$, showing the stability and controlled release of the CLPNs. These results could be explained as the lipid shell of CLPNs has high affinity to the lipid-structured cell surface, the cationic surface charge could absorb onto the negatively charged cell surface, promote the fusion of the nanocarriers to the cell membrane and deliver more drug and gene into the tumor cells (39). The structure of CLPNs may delay the drug/gene release more than other vectors, bring about the long-lasting drug/gene delivery effect in tumor tissues (40). Outstanding delivery ability of CLPNs for both CDDP and DNA could combine the therapeutic efficiency of both drug and gene for the treatment of pediatric RMS.

In the present study, we described a simple and efficient method for the fabrication of CLPNs for co-delivery of CDDP and DNA for the therapy of childhood head and neck cancers. The results illustrated that the concentration of the cationic lipid has influence on the characteristics of CLPNs. In vitro anticancer effect, in vitro transfection efficiency, in vivo antitumor and gene delivery efficacy of CDDP/DNA CLPNs have advantages over other formulations tested. Excellent ability of CLPNs for co-delivery of CDDP and DNA could combine the therapeutic efficiency of both drug and gene for the treatment of pediatric RMS.

\section{References}

1. Ward E, DeSantis C, Robbins A, Kohler B and Jemal A: Childhood and adolescent cancer statistics, 2014. CA Cancer J Clin 64: 83-103, 2014.

2. Choi DK and Schmidt ML: Chemotherapy in children with head and neck cancers: Perspectives and review of current therapies. Oral Maxillofacial Surg Clin N Am 28: 127-138, 2016.

3. Marcus KJ and Tishler RB: Head and neck carcinomas across the age spectrum: Epidemiology, therapy, and late effects. Semin Radiat Oncol 20: 52-57, 2010.

4. Wang Y, Lipari P, Wang X, Hailey J, Liang L, Ramos R, Liu M, Pachter JA, Bishop WR and Wang Y: A fully human insulin-like growth factor-I receptor antibody SCH 717454 (Robatumumab) has antitumor activity as a single agent and in combination with cytotoxics in pediatric tumor xenografts. Mol Cancer Ther 9: 410-418, 2010.

5. Morrison R, Gardiner C, Evidente A, Kiss R and Townley H: Incorporation of ophiobolin a into novel chemoembolization particles for cancer cell treatment. Pharm Res 31: 2904-2917, 2014.

6. Xie M, Zhang H, Xu Y, Liu T, Chen S, Wang J and Zhang T: Expression of folate receptors in nasopharyngeal and laryngeal carcinoma and folate receptor-mediated endocytosis by molecular targeted nanomedicine. Int J Nanomed 8: 2443-2451, 2013.

7. Chen Y, Xu G, Zheng Y, Yan M, Li Z, Zhou Y, Mei L and Li X: Nanoformulation of D- $\alpha$-tocopheryl polyethylene glycol 1000 succinate-b-poly( $\varepsilon$-caprolactone-ran-glycolide) diblock copolymer for siRNA targeting HIF-1 $\alpha$ for nasopharyngeal carcinoma therapy. Int J Nanomed 10: 1375-1386, 2015.

8. Koganti S, Jagani HV, Palanimuthu VR, Mathew JA, Rao MC and Rao JV: In vitro and in vivo evaluation of the efficacy of nanoformulation of siRNA as an adjuvant to improve the anticancer potential of cisplatin. Exp Mol Pathol 94: 137-147, 2013.

9. Oberoi HS, Nukolova NV, Kabanov AV and Bronich TK: Nanocarriers for delivery of platinum anticancer drugs. Adv Drug Deliv Rev 65: 1667-1685, 2013.

10. Kojima H, Iida M, Yaguchi Y, Suzuki R, Hayashi N, Moriyama H and Manome Y: Enhancement of cisplatin sensitivity in squamous cell carcinoma of the head and neck transfected with a survivin antisense gene. Arch Otolaryngol Head Neck Surg 132: 682-685, 2006.

11. Jiang M, Liu Z, Xiang Y, Ma H, Liu S, Liu Y and Zheng D: Synergistic antitumor effect of AAV-mediated TRAIL expression combined with cisplatin on head and neck squamous cell carcinoma. BMC Cancer 11: 54, 2011. 
12. Shao Z, Shao J, Tan B, Guan S, Liu Z, Zhao Z, He F and Zhao J: Targeted lung cancer therapy: Preparation and optimization of transferrin-decorated nanostructured lipid carriers as novel nanomedicine for co-delivery of anticancer drugs and DNA. Int J Nanomed 10: 1223-1233, 2015.

13. Teo PY, Cheng W, Hedrick JL and Yang YY: Co-delivery of drugs and plasmid DNA for cancer therapy. Adv Drug Deliv Rev 98: 41-63, 2016

14. Yang T, Zhao P, Rong Z, Li B, Xue H, You J, He C, Li W, He X, Lee RJ, et al: Anti-tumor efficiency of lipid-coated cisplatin nanoparticles co-loaded with microRNA-375. Theranostics 6: 142-154, 2016.

15. Wasungu L and Hoekstra D: Cationic lipids, lipoplexes and intracellular delivery of genes. J Control Release 116: 255-264, 2006.

16. Zhang L, Chan JM, Gu FX, Rhee JW, Wang AZ, Radovic-Moreno AF, Alexis F, Langer R and Farokhzad OC: Self-assembled lipid - polymer hybrid nanoparticles: A robust drug delivery platform. ACS Nano 2: 1696-1702, 2008

17. Yang XZ, Dou S, Wang YC, Long HY, Xiong MH, Mao CQ, Yao YD and Wang J: Single-step assembly of cationic lipid-polymer hybrid nanoparticles for systemic delivery of siRNA. ACS Nano 6: 4955-4965, 2012.

18. Bose RJ, Arai Y, Ahn JC, Park H and Lee SH: Influence of cationic lipid concentration on properties of lipid-polymer hybrid nanospheres for gene delivery. Int J Nanomed 10: 5367-5382, 2015.

19. He C, Lu J and Lin W: Hybrid nanoparticles for combination therapy of cancer. J Control Release 219: 224-236, 2015.

20. Hadinoto K, Sundaresan A and Cheow WS: Lipid-polymer hybrid nanoparticles as a new generation therapeutic delivery platform: A review. Eur J Pharm Biopharm 85 (3 Pt A): 427-443, 2013.

21. Guo S, Wang Y, Miao L, Xu Z, Lin CM, Zhang Y and Huang L: Lipid-coated Cisplatin nanoparticles induce neighboring effect and exhibit enhanced anticancer efficacy. ACS Nano 7: 9896-9904, 2013.

22. Han L, Ren Y, Long L, Zhong Y, Shen C, Pu P, Yuan X and Kang C: Inhibition of C6 glioma in vivo by combination chemotherapy of implantation of polymer wafer and intracarotid perfusion of transferrin-decorated nanoparticles. Oncol Rep 27: 121-128, 2012.

23. Nakata H, Miyazaki T, Iwasaki T, Nakamura A, Kidani T, Sakayama K, Masumoto J and Miura H: Development of tumor-specific caffeine-potentiated chemotherapy using a novel drug delivery system with Span 80 nano-vesicles. Oncol Rep 33: 1593-1598, 2015

24. Yu W, Liu C, Ye J, Zou W, Zhang N and Xu W: Novel cationic SLN containing a synthesized single-tailed lipid as a modifier for gene delivery. Nanotechnology 20: 215102, 2009.

25. Wang RH, Cao HM, Tian ZJ, Jin B, Wang Q, Ma $\mathrm{H}$ and Wu J: Efficacy of dual-functional liposomes containing paclitaxel for treatment of lung cancer. Oncol Rep 33: 783-791, 2015.

26. Yu W, Liu C, Liu Y, Zhang N and Xu W: Mannan-modified solid lipid nanoparticles for targeted gene delivery to alveolar macrophages. Pharm Res 27: 1584-1596, 2010.
27. Yang Q, Zhang S, Kang M, Dong R and Zhao J: Synergistic growth inhibition by sorafenib and cisplatin in human osteosarcoma cells. Oncol Rep 33: 2537-2544, 2015.

28. Almeida PV, Shahbazi MA, Mäkilä E, Kaasalainen M, Salonen J, Hirvonen J and Santos HA: Amine-modified hyaluronic acid-functionalized porous silicon nanoparticles for targeting breast cancer tumors. Nanoscale 6: 10377-10387, 2014.

29. Ye Y, Zhang X, Zhang T, Wang H and Wu B: Design and evaluation of injectable niclosamide nanocrystals prepared by wet media milling technique. Drug Dev Ind Pharm 41: 1416-1424, 2015.

30. Gao L, Xu Z, Wang Y, Sun B, Song Z, Yang B, Liu X, Lin Y, Peng J, Han G, et al: Anticancer effect of SZC017, a novel derivative of oleanolic acid, on human gastric cancer cells. Oncol Rep 35: 1101-1108, 2016.

31. Lv X, Liu F, Shang Y and Chen SZ: Honokiol exhibits enhanced antitumor effects with chloroquine by inducing cell death and inhibiting autophagy in human non-small cell lung cancer cells. Oncol Rep 34: 1289-1300, 2015.

32. Mainardes RM and Evangelista RC: PLGA nanoparticles containing praziquantel: Effect of formulation variables on size distribution. Int J Pharm 290: 137-144, 2005.

33. Liu P, Sun L, Zhou DS, Zhang P, Wang YH, Li D, Li QH and Feng RJ: Development of alendronate-conjugated poly (lactic-co-glycolic acid)-dextran nanoparticles for active targeting of cisplatin in osteosarcoma. Sci Rep 5: 17387, 2015.

34. Asthana S, Jaiswal AK, Gupta PK, Dube A and Chourasia MK: Th-1 biased immunomodulation and synergistic antileishmanial activity of stable cationic lipid-polymer hybrid nanoparticle: Biodistribution and toxicity assessment of encapsulated amphotericin B. Eur J Pharm Biopharm 89: 62-73, 2015.

35. Valencia PM, Basto PA, Zhang L, Rhee M, Langer R, Farokhzad OC and Karnik R: Single-step assembly of homogenous lipid-polymeric and lipid-quantum dot nanoparticles enabled by microfluidic rapid mixing. ACS Nano 4: 1671-1679, 2010

36. Seedat N, Kalhapure RS, Mocktar C, Vepuri S, Jadhav M, Soliman M and Govender T: Co-encapsulation of multi-lipids and polymers enhances the performance of vancomycin in lipid-polymer hybrid nanoparticles: In vitro and in silico studies. Mater Sci Eng C 61: 616-630, 2016.

37. D'Mello S, Salem AK, Hong L and Elangovan S: Characterization and evaluation of the efficacy of cationic complex mediated plasmid DNA delivery in human embryonic palatal mesenchyme cells. J Tissue Eng Regen Med 10: 927-937, 2016.

38. Dong W, Jin GH, Li SF, Sun QM, Ma DY and Hua ZC: Cross-linked polyethylenimine as potential DNA vector for gene delivery with high efficiency and low cytotoxicity. Acta Biochim Biophys Sin (Shanghai) 38: 780-787, 2006.

39. Khurana B, Goyal AK, Budhiraja A, Aora D and Vyas SP. Lipoplexes versus nanoparticles: pDNA/siRNA delivery. Drug Deliv 20: 57-64, 2013.

40. Krishnamurthy S, Vaiyapuri R, Zhang L and Chan JM: Lipid-coated polymeric nanoparticles for cancer drug delivery. Biomater Sci 3: 923-936, 2015. 